Instituto Internacional de Investigación y Desarrollo Tecnológico Educativo INDTEC, C.A.

DOI: https://doi.org/10.29394/Scientific.issn.2542-2987.2018.3.7.16.309-328

OAI-PMH: http://www.indteca.com/ojs/index.php/Revista Scientific/oai

\title{
Fortalecimiento de la Educación Ambiental a Través de la Siembra de Plantas Ornamentales
}

Autora: Yosmary Del Valle Mendoza Universidad Pedagógica Experimental Libertador, UPEL yos14mendoza@gmail.com Barinas, Venezuela

\section{Resumen}

El propósito del presente trabajo de investigación es fortalecer la educación ambiental a través de la siembra de plantas ornamentales en el Sector 3 de Mayo. En Ciudad Bolivia, Municipio Pedraza, Estado Barinas. Este trabajo se enmarcó el paradigma cualitativo apoyado en una investigación acción. Los informantes claves estuvieron representados en tres habitantes del sector 3 de mayo que con la característica de ser miembros activos del Consejo Comunal de la localidad: (1) uno perteneciente al comité de educación, (1) uno que hace parte al comité de tierra urbana y rural y (1) uno integrante del comité de salud. Se determinó que los habitantes de la comunidad no propician actividades de embellecimiento y sensibilización ambiental. La técnica de recolección de información utilizada fue la entrevista, aplicando un guion contentivo de (5) cinco interrogantes de característica abierta relacionadas con la temática abordada. Dentro de los testimonios de los informantes se encontraron los siguientes hallazgos, los entrevistados manifestaron que tienen escasez de formación en relación con el tema de ornato y mantenimiento de áreas verdes. Situación que requiere del desarrollo de un plan de acción de ornato y arborización para fortalecer la educación ambiental en la colectividad.

Palabras clave: educación ambiental; planta; recursos naturales. 


\title{
Strengthening of Environmental Education Through the Planting of Ornamental Plants
}

\begin{abstract}
The purpose of this research work is to develop ornamentation and afforestation activities to strengthen Environmental Education in the Sector 3 de Mayo. In Ciudad Bolivia, Pedraza Municipality, Barinas State. This work framed the qualitative paradigm supported by an action research. The key informants were represented in three May 3 sector inhabitants who with the characteristic of being active members of the Local Council of the locality: (1) one belonging to the education committee, (1) one that is part of the urban land committee and rural and (1) one member of the health committee. It was determined that the inhabitants of the community do not encourage activities of beautification and environmental awareness. The technique used to collect information was the interview, applying a contention script of (5) five openended questions related to the subject matter. Within the informants' testimonies the following findings were found, interviewees said that they lack training in relation to the subject of ornamentation and maintenance of green areas. Situation that requires the development of an action plan of ornamentation and afforestation to strengthen environmental education in the community.
\end{abstract}

Keywords: environmental education; plants; natural resources.

Date Received: 13-07-2017

Date Acceptance: 07-10-2017 


\section{Introducción}

En la actualidad los problemas ambientales se derivan del deterioro de los recursos naturales; es el ambiente quien provee al ser humano de lo necesario para su desenvolvimiento sobre el planeta, por lo tanto, es el individuo quien debería darle un tratamiento adecuado, sin embargo, los proyectos que buscan el desarrollo de una nación causan un impacto sustancialmente en él. Lo que ocasiona que donde existió un bosque hoy exista asfalto y donde antes estuvo una laguna hoy este presente un vertedero de basura. Barros, (2013a), afirma que "Este proceso se acelerará en las próximas décadas; si no se produce un cambio en el comportamiento de la humanidad, las consecuencias serán catastróficas durante el siglo XXIl" (pág. $5)$.

Según Barros, (2013b), "El cambio climático aparece como un problema ético, de responsabilidad y solidaridad hacia las futuras generaciones y la especie humana e incluso hacia la vida en el resto del planeta" (pág. 13). Para contribuir a la conservación del ambiente es necesario realizar un esfuerzo para que cada individuo alcance un grado de conciencia acerca de lo necesario que es para sí mismo el ambiente.

Fernández, (2014a), expresa que "la explosión demográfica; la conciencia, el desarrollo económico no siempre ha supuesto beneficios para la humanidad ha desencadenado alteraciones ecológicas de grave consecuencia, la previsión de un futuro incierto con enormes problemas ambientales, y agotamiento de los recursos naturales" (pág. 31). Indudablemente que el crecimiento de la población trae consigo algunos perjuicios a la naturaleza, asimismo la acumulación de desechos en sitios inapropiados, deforestación, aire contaminado, que adicionalmente trasciende en la salud de las comunidades.

En base a lo expuesto anteriormente, esta investigación tuvo como propósito general, fortalecer la educación ambiental a través de la siembra de 
plantas ornamentales se desarrolla en función de diagnosticar las necesidades de formación ambiental a partir del avance de un plan acción de ornato y arborización en el Sector 3 de Mayo. En Ciudad Bolivia, Municipio Pedraza, Estado Barinas.

\section{El problema}

La temática ambiental en la actualidad es una preocupación para toda la humanidad, la contaminación en el mundo ha tomado grandes dimensiones, lo cual ha llamado la atención de los gobernantes de naciones por las enormes cantidades de recursos que se deben invertir en la recolección y procesamiento de residuos de origen doméstico y de origen industrial.

En este sentido, la Agenda 21, establecida en la Cumbre Mundial realizada en Río de Janeiro por la Organización de las Naciones Unidas sobre el Ambiente y Desarrollo, fue propuesta como principal plan mundial para enfrentar problemas económicos y ecológicos del siglo XX. Sitarz, (1993), citado por Nebel y Wright, (2013), ha definido la Agenda 21, como "un documento de esperanza para la solución a los problemas ambientales y económicos del mundo". Desde este punto de vista los Estados deben estar ejecutando esta propuesta y que inquiere soslayar tanta contaminación de los países desarrollados.

Una de las conclusiones de esta Agenda 21, es la exhortación a los diferentes gobiernos a preparar o actualizar estrategias orientadas al logro de la integración ambiente y desarrollo de manera que permita aumentar la capacidad de las poblaciones para abordar asuntos ambientales sin ocasionarle detrimento, estableciendo además, la orientación a los Estados de proteger el ambiente como recurso no renovable, el cual esta vulnerable ante la mano del hombre que lo deteriora.

Así mismo, el Protocolo de Kyoto, realizado en Montreal en 1997, citado por Informe 21 (2013), plantea la preservación de la vida humana, así mismo 
la vegetación y los animales, estableciendo acuerdos de disminución y control, sobre las emisiones de gases y contaminantes que degraden el ambiente, dichos acuerdos están fijados por países que emiten mayores contaminantes y con este acuerdo se comprometen a disminuir la degradación del ambiente. Así como también, lo relativo a las sustancias que agotan la capa de ozono, protección y mejora de los sumideros y depósitos de los gases de efecto invernadero no controlados por el Protocolo de Montreal, tomando en cuenta los compromisos en virtud de los acuerdos internacionales pertinentes sobre el ambiente; promoción de prácticas sostenibles de gestión forestal y la reforestación.

Para Hitcher, (2013), "los seres humanos están llamados a un cambio radical de conciencia, pues con la defensa de los recursos hídricos, los ecosistemas, la biodiversidad y un modelo de desarrollo sustentable se garantiza la continuidad de la humanidad" (pág. 55). Cada vez que ocurre una agresión hacia el ambiente se pone en peligro la madre Tierra. No se trata de poner al individuo como un ser depredador, tiene que ver con los valores en la sociedad y los modos de vida cada vez son más ostentosos.

Fernández, (2014b), Afirma "hace falta una mayor conciencia para cuidar cada espacio ambiental el modelo desarrollista amenaza con destruir nuestro planeta" (pág. 144). Esta afirmación describe como un modelo de políticas empleadas en la comercialización e industrialización ha traído la debacle para diversos ecosistemas, al mismo tiempo el menoscabo de los procesos participativos y la toma de decisiones de los pueblos hacia una educación ambiental coherente, para la conservación de los recursos naturales.

Así mismo la Organización no gubernamental denominada Red Voltaire, expresa que en Brasil, en la ciudad de Curitiba (2006), se realizó el Manifiesto de las Américas en defensa de la naturaleza y de la diversidad biológica y cultural, donde una de sus conclusiones fue la necesidad "de pasar 
de una sociedad de producción industrial, consumista e individualista, que lesiona los ecosistemas a una sociedad que logre la sustentación de toda la existencia, que se oriente por un modo socialmente justo y ecológicamente sustentable." (pág. 5)

Por otro lado, Sepúlveda (2013), asevera que "la agricultura, los recursos naturales y desarrollo rural, son actividades productivas y económicas se relacionan con el deterioro de los recursos naturales, poniéndolo en el centro" (pág. 28). Además, afirma que el desarrollo depende de la capacidad que tengan los actores comunitarios para manejar los recursos económicos en armonía con el potencial de los recursos naturales.

Para León (2013), "en Venezuela es una realidad la existencia de problemas ambientales" (pág. 4). Esto se remonta a las políticas ambientales aplicadas por parte del Estado que basaba su razón de ser, en la búsqueda del desarrollo económico, sin importar irremediablemente a una expoliación de los factores ambientales y una degradación de los ecosistemas. Disminuyendo los principios ambientales y sacrificio de los valores ecológicos.

En Barinas paralelamente las condiciones ambientales, han generado un efecto opuesto al equilibrio entre el entorno natural y el avance poblacional; trayendo como consecuencias, la disminución de caudales en los ríos y quebradas. Del mismo modo la inhalación de aire contaminante, aplicación de tala y quema, mermas en algunas especies de flora y fauna, deterioro de las áreas verdes en las comunidades, han desaparecido importantes zonas aptas para la observación de estas especies autóctonas.

En tal sentido Martínez (2013), Señala "la necesidad de contemplar de forma explícita el ambiente en los procesos educativos" (pág. 5), los procesos de educación ambiental aportan nuevos modelos educativos y profundizan en una reorientación de la educación impartida hacia la conservación y preservación de los recursos naturales. El papel de la educación debe ser encaminado a la solución de los problemas ambientales, sin dejar a un lado la 
participación de las comunidades en el proceso, al mismo tiempo la interrelación entre ser humano en armonía con la naturaleza. Es por ello que la incorporación de la educación ambiental constituye una actitud responsable y comprometida ante el ambiente.

Si no se educa a la población, acerca del peligro que representa el seguir deprimiendo irresponsablemente los recursos naturales y el ambiente, en poco tiempo el ser humano se estará lamentando más sobre las situaciones que afecten a los seres vivos. Por otro lado, es significativo un proceso de participación que vaya dirigido a las comunidades esencialmente en el área de la conservación del ambiente enfocado en el ornato y mantenimiento de sus comunidades.

Asimismo, Montaldo (2013), sostiene que "la educación realiza el mantenimiento y transmisión de la cultura, a fin de conseguir su continuidad" (pág. 3). La promoción de la educación ambiental tiene alcances importantes y puede llevar al desarrollo de una comunidad permitiendo que se originen nuevas destrezas y resuelvan los problemas ambientales.

La comunidad donde se realiza la investigación reúne las características favorables para el estudio, porque en ella existe abandono de áreas verdes, mala colocación de los desechos sólidos en los patios traseros y frentes de las casas, vertido de aguas residuales de auto lavados improvisados en las casas, entre otros.

La problemática antes planteada, permite señalar que en dicha comunidad existe una deficiente formación ambiental lo que afecta la calidad ambiental de la misma, además de provocar efectos sobre la salud de la población, sin percatarse los habitantes de la misma, que tal situación mayormente es ocasionada por escasa sensibilización y conocimiento sobre valores ambientales. Por ello, es necesario crear los mecanismos que permitan plantear acciones dirigidas a la formación de las comunidades en materia educativa ambiental para mejorar la calidad de su entorno. 
En este sentido, esta investigación tiene la finalidad de desarrollar actividades ambientales con la participación de la comunidad para el ornato y la arborización en el Sector 3 de Mayo. Parroquia Ciudad Bolivia, Municipio Pedraza, del Estado Barinas. Por lo antes planteado, se propone dar respuesta a las siguientes interrogantes:

¿Cuál es la necesidad de desarrollar actividades ambientales con la participación de la comunidad para su ornato y arborización?, ¿Se podrá diseñar un plan de acción para ornato y arborización de la comunidad?, ¿Qué actividades se podrán desarrollar para el ornato y arborización del sector 3 de mayo?, ¿Qué resultados se obtendrían del desarrollo del plan de acción para el ornato y arborización del sector 3 de mayo?

\section{Referentes Teóricos}

\subsection{Constructivismo}

Esta investigación está orientada en el marco de la corriente constructivista, según Chadwick (1999), "la esencia del constructivismo es el individuo como construcción propia que se va produciendo como resultado de la interacción de sus disposiciones internas y su medio ambiente" (pág. 464), y su conocimiento no es una copia de la realidad, sino un construcción de la persona misma, puesto que se da a través de los procesos de aprendizaje que el estudiante va construyendo en estructuras, es decir, las diversas formas de organizar la información, las cuales facilitarán mucho el futuro aprendizaje, porque son las representaciones organizadas de experiencia previa, relativamente permanentes y sirven como esquemas que funcionan para filtrar, codificar, categorizar y evaluar la información que el estudiante recibe en relación con alguna experiencia relevante.

\subsection{Ornato}

El término ornato dentro de este estudio, hace referencia hacia el 
mantenimiento y cuidados del medio biótico existente, es decir de las áreas verdes y especies vegetales en espacios públicos. Para Zapata (2006), el ornato es definido como "embellecimiento de espacios naturales y arquitectónicos con la finalidad de entrelazar estos elementos al objeto de obtener una composición equilibrada entre lo natural y lo artificial" (pág. 1). Por lo tanto, las áreas verdes han sido incorporadas a las ciudades para devolverle al entorno urbano los espacios naturales que se han perdido.

\section{3. plantas ornamentales}

Las plantas representan un atractivo a vista de los seres humanos lo cual ofrece un ambiente agradable, en este sentido la arborización es importante para recuperar espacios naturales intervenidos por la acción del hombre ofreciéndoles a las personas que viven en centros urbanos disfrutar de la naturaleza. Las plantas ornamentales se utilizan para ornamentar los espacios poblados, según Sánchez (2012);

Se clasifica en tres grandes grupos: plantas de vivero (grupo 1), utilizadas siempre al exterior en jardines, siendo cultivadas normalmente en plena tierra; plantas de floristas (grupo 2), utilizadas como flor cortada o verde de acompañamiento en arreglos y composiciones florales; y plantas de interior (grupo 3 ), utilizadas mayormente en la decoración de interiores, siendo cultivadas en macetas y jardineras. (pág. 10).

Aunque con diferentes usos, todas ellas basan su atractivo en cualidades estéticas, tales como el color, la textura, el porte o la forma. Las plantas ornamentales se pueden considerar como aquellas plantas o sus partes que en su estado natural o preservadas pueden cubrir la función de satisfacer visualmente el gusto del consumidor y en algunos casos presentar un atractivo en sus flores, frutos o de tipo aromático. Asimismo, Contreras (2015), Expone:

La conservación implica necesariamente un conocimiento de la 
ecología, la ciencia que concierne a las relaciones entre la vida y ambiente, pero la ecología misma se basa en una amplia variedad de disciplinas, y la conservación abarca sentimientos, creencias y actitudes a veces complementarias y otras divergentes entre ciencia y tecnología. (pág. 80).

Razón por la cual este trabajo investigativo orienta al ornato en función de la conservación del ambiente, ofreciéndole a las personas sujetos de investigación conocimientos esenciales de ecología, los cuales son importantes para mejorar la relación entre el hombre y el ambiente, con respecto a la comunidad donde se desarrolla el estudio la nueva experiencia contribuye con el mejor manejo de sus áreas verde.

\subsection{Educación ambiental}

La educación ambiental es fundamental para la formación de los ciudadanos con el fin de construir una cultura ecológica que mejore la relación del hombre con el ambiente, según Urrea (2016), "Se trata de un contexto en el que se desarrolla una toma de conciencia en torno a las condiciones del ambiente y la manera de definir el impacto del ser humano y su sociedad". (pág. 158). De acuerdo con esta definición la educación debe estar orientada a la sensibilización de los seres humanos en la preservación del planeta.

\section{Marco Metodológico}

El estudio está enmarcado dentro de los parámetros de la investigación acción, por cuanto se trata de resolver un problema real concreto, sin llegar a la generalización teórica, con el objeto de mejorar la práctica. Por lo tanto, el problema que motiva este trabajo se ubica dentro de la investigación acción participante. La investigación acción se realiza en varias etapas a saber: EI diseño de una propuesta de cambio se realiza después del análisis e interpretación de la información recopilada y siempre a la luz de los objetivos 
que se persiguen, se está en condiciones de visualizar el sentido de los mejoramientos que se desean. (Hurtado y Toro, 2005a, pág. 35).

Los pasos o fases de la investigación acción participante, según Hurtado y Toro (2005b), son:

Fase I: Problematización. Considerando que la labor educativa se desarrolla en situaciones donde se presentan problemas prácticos. Lo lógico es que un proyecto de este tipo comience a partir de un problema práctico: en general, se trata de incoherencias o inconsistencias entre lo que se persigue y los que en la realidad ocurre. Es posible diferenciar entre: contradicciones, cuando existe oposición entre la formulación de las pretensiones del autor, por una parte, y sus actuaciones por otro. Dilemas, un tipo especial de contradicción, pudiendo presentarse como dos tendencias irreconciliables que se descubren al analizar la práctica, pero que revelan valores necesarios, o bien diferencias de interese o motivaciones entre dos o más partes. Dificultades o limitaciones, aquellas situaciones en que se encuentran ante la oposición para desarrollar las actuaciones deseables de instancias que no se pueden modificar o influir desde la actuación directa e inmediata, lo cual requeriría una actuación a largo plazo, como es el caso de ciertas inercias institucionales o formas de organización.

Fase II: Diagnostico: una vez que se ha identificado el significado del problema que será el centro del proceso de investigación, y habiendo formulado un enunciado del mismo, es necesario realizar la recopilación de información que permitirá un diagnóstico claro de la situación. La búsqueda de información consiste en recoger diversas evidencias que permitan una reflexión a partir de una mayor cantidad de datos. Esta recopilación de información debe expresar el punto de vista de las personas implicadas, informar introspectivamente sobre las personas implicadas, es decir, como viven y entienden la situación que se investiga. En síntesis, al análisis reflexivo 
que lleva a una correcta formulación del problema y a la recopilación de información necesaria para un buen diagnóstico, representa al camino hacia el planteamiento de líneas de acción coherentes relacionadas con el problema de ornato y arborización que se presenta en la comunidad 3 de mayo.

Fase III: Diseño de actividades: una vez que se ha realizado el análisis e interpretación de la información recopilada y siempre a la luz de los propósitos de la investigación que se persiguen, se está en condiciones de visualizar el sentido de los mejoramientos que se desean. La reflexión, que en este caso se vuelve prospectiva, es la que permite llegar a diseñar una propuesta de cambio y mejoramiento, para la situación que ocurre en la Comunidad 3 de Mayo en relación al ornato y arborización del sector. Del mismo modo, es necesario en este momento definir un diseño de evaluación de la misma. Es decir, anticipar los indicadores y metas que darán cuenta del logro de las actividades.

Fase IV: Aplicación de las actividades: una vez diseñada la propuesta de acción, esta es llevada a cabo por las personas interesadas. Es importante, sin embargo, comprender que cualquier propuesta a la que se llegue tras este análisis y reflexión, debe ser entendida en un sentido hipotético, es decir, se emprende una nueva forma de actuar, un esfuerzo de innovación y mejoramiento de la práctica que debe ser sometida permanentemente a condiciones de análisis, evaluación y reflexión.

Fase V: Evaluación: todo este proceso, que comenzaría otro ciclo en la espiral de la investigación - acción, va proporcionando evidencias del alcance y las consecuencias de las acciones emprendidas, y de su valor como mejora de la práctica.

\subsection{Diseño de la Investigación}

El estudio constituye un estudio de tipo cualitativo. El diseño de esta investigación es de campo porque los datos se obtienen directamente de la 
realidad, de esta manera se estudia en profundidad la Comunidad 3 de Mayo para el ornato y arborización de la misma y así fortalecer la educación ambiental. Al respecto Bavaresco (2013), afirma que el diseño de campo: "consiste en la caracterización de un hecho, fenómeno o grupo con el fin de establecer su estructura o su comportamiento". (pág. 48).

\subsection{Escenario e informantes claves}

La investigación se llevó a cabo en la Comunidad 3 de Mayo, ubicada en Ciudad Bolivia capital del municipio Pedraza del estado Barinas, pertenece al ámbito urbano de la población. Los actores sociales contentivos en este estudio se refieren a la población, de donde se extraen los informantes claves para el respectivo estudio. Los cuales están representados en tres habitantes del sector 3 de mayo que con la característica de ser miembros activos del Consejo Comunal de la localidad: (1) uno perteneciente al comité de educación, (1) uno que hace parte al comité de tierra urbana y rural y (1) uno integrante del comité de salud. Los testimonios de estas personas son fundamentales para lograr los propósitos de la investigación

\subsection{Técnicas e instrumentos de recolección de información}

La técnica de recolección utilizada para esta investigación es la entrevista. Para Hurtado y Toro (2005c), "recoger datos es reducir de modo intencionado y sistemático mediante el empleo de los sentidos o de un instrumento mediador la realidad natural y compleja que se pretende estudiar" (pág. 142).

Es importante señalar, que la entrevista es una técnica apropiada para las investigaciones cualitativas de tal modo que se concreta en un instrumento por el cual una persona invita, cara a cara a otra para que le facilite una información, esta se puede basar desde una conversación libre hasta una interrogación estructurada. Tamayo y Tamayo (2013a, pág. 101), la definen 
como "reiterados encuentros cara a cara entre el investigador y los informantes, encuentros dirigidos a la comprensión de las perspectivas que tienen los informantes de sus experiencias o situaciones tal como las expresan con sus propias palabras".

En este mismo orden de ideas, se tiene que, para la recolección de la información, se desarrollan las entrevistas, tomando en consideración un inicio, un desarrollo y un cierre. Es importante señalar que, con la aplicación de esta técnica, se obtiene la información necesaria por parte de los informantes claves. Asimismo, se utiliza la técnica de observación para contrastar las respuestas de la entrevista realizadas a los miembros del Consejo Comunal de 3 de Mayo, el instrumento que acompaña a la técnica de observación se llama registro escrito, en el cual se lleva el control de los testimonios dados por las unidades de análisis.

\subsection{Validación}

La validez y fiabilidad de un instrumento de la investigación es sumamente importante porque la misma arrojará datos reales que pueden generarse en la elaboración de teorías, por lo tanto, dentro de esta investigación la validez se está enfocada en lo que define Tamayo y Tamayo (2013b), "eficacia con que un instrumento mide lo que se pretende" (pág. 193). De tal manera que el mismo se basa en el juicio de expertos en donde el instrumento es sometido a la revisión de profesionales con especialidad en Educación Ambiental y Metodología de la Investigación, quienes determinan si el instrumento en cuestión presenta las condiciones de validez de contenido.

Para la fiabilidad, los elementos se sustentan en una acción de tipo participativa e interactiva en donde la investigadora aplicará los instrumento a personas de otra comunidad que tengan las mismas características del contexto que se estudia, para posteriormente elaborar el análisis con los propios entrevistados y así verificar la pertinencia de los aspectos presentes 
en el guion de la entrevista y así se puede aplicar la entrevista en forma de ensayo a cinco miembros de otra comunidad que presente las mismas características de la comunidad objeto de estudio.

Con la aplicación de la entrevista y las observaciones realizadas por parte de la investigadora a los informantes claves, se tiene que ellos tienen una definición de las plantas ornamentales, pero no significan personalmente nada para ellos, puesto que no le dan la importancia a la misma en cuanto a tener un jardín en frente de sus casas; tampoco la comunidad tiene áreas verdes, sin embargo, existen espacios desolados que pudieran arborizarse.

En cuanto al mantenimiento que ellos pudieran realizar las áreas verdes existentes en la comunidad, el diagnóstico arrojó que un solo informante clave se encarga de conservar limpio al menos el patio de su casa, realizando acciones de mantenimiento al mismo, otro directamente no lo hace, pero ejecuta acciones para que otros realicen jornadas de limpiezas a terrenos baldíos, pero hay uno que no ejecuta ninguna acción. Por eso el sector 3 de mayo presenta problemas de acumulación que le resta estética sobre el ornato y arborización de la comunidad.

Asimismo, los miembros del consejo comunal no propician actividades de embellecimiento para el sector, jornadas de sensibilización ambiental, de ornato, arborización y embellecimiento, creando jardines con plantas ornamentales autóctonas, medicinales, que favorezcan el embellecimiento de la comunidad. Hay escasez de formación en relación con el tema de ornato y mantenimiento de áreas verdes, por parte de los miembros del Consejo Comunal.

\subsection{Análisis de la información}

La recolección de la información se lleva a cabo a través de las entrevistas realizadas a los sujetos objeto de estudio, indicándoles la necesidad de que sus respuestas sean veraces, así como también el registro 
diario de las observaciones que se le realizaran a los mismos, con la finalidad de indagar los conocimientos que estos poseen con relación a las áreas verdes, mantenimiento y plantas. Para luego llevar a cabo el proceso de organización y categorización de la información, y finalmente interpretar y discutir los resultados, para la elaboración de la reflexión final.

Con la aplicación de la entrevista y las observaciones realizadas por parte de la investigadora a los informantes claves, se tiene que ellos tienen una definición de las plantas ornamentales, pero no significan personalmente nada para ellos, puesto que no le dan la importancia a la misma en cuanto a tener un jardín en frente de sus casas; tampoco la comunidad tiene áreas verdes, sin embargo, existen espacios desolados que pudieran arborizarse.

En cuanto al mantenimiento que ellos pudieran realizar las áreas verdes existentes en la comunidad, el diagnóstico arrojó que un solo informante clave se encarga de conservar limpio al menos el patio de su casa, realizando acciones de mantenimiento al mismo, otro directamente no lo hace, pero ejecuta acciones para que otros realicen jornadas de limpiezas a terrenos baldíos, pero hay uno que no ejecuta ninguna acción. Por eso el sector 3 de mayo presenta problemas de acumulación que le resta estética sobre el ornato y arborización de la comunidad.

Asimismo, los miembros del consejo comunal no propician actividades de embellecimiento para el sector, jornadas de sensibilización ambiental, de ornato, arborización y embellecimiento, creando jardines con plantas ornamentales autóctonas, medicinales, que favorezcan el embellecimiento de la comunidad. Hay escasez de formación en relación el tema de ornato y mantenimiento de áreas verdes, por parte de los miembros del Consejo Comunal.

Aunado a esto, se tiene que en este sector no se realizan actividades de participación e integración con la comunidad para generar acciones que favorezcan el ambiente, quizás por eso es que la comunidad en materia 
ambiental esta grave. De tal manera que este diagnóstico genera como posible solución la necesidad de desarrollar actividades de ornato y arborización para el fortalecimiento de la educación ambiental.

\section{Conclusiones}

Esta investigación demostró que hay una necesidad del diseño de actividades ambientalistas para el fortalecimiento de la Educación Ambiental en el Sector 3 de Mayo. En Ciudad Bolivia, Municipio Pedraza, Estado Barinas, porque los miembros del consejo comunal tienen una definición de las plantas ornamentales, pero para ellos no tienen ningún significado, puesto que no le dan la importancia a la misma en cuanto a tener un jardín en frente de sus casas; del mismo modo en la comunidad no hay áreas verdes, viéndose de manera desolada, sin ninguna riqueza ambiental.

Por ende, los miembros del consejo comunal no propician actividades de embellecimiento para el sector, jornadas de sensibilización ambiental, de ornato, arborización y embellecimiento, creando jardines con plantas ornamentales autóctonas, medicinales, que favorezcan el embellecimiento de la comunidad, porque hay escasez de formación en relación con el tema de ornato y mantenimiento de áreas verdes, por parte de los miembros del Consejo Comunal y por consiguiente en la comunidad en general.

Se desarrollaron una serie de charlas y encuentros que propiciaron la integración, participación y planificación de acciones para la realización de jornadas de ornato y embellecimiento del sector, con la participación de los miembros del consejo comunal y los vecinos del sector, en donde ellos buscaron soluciones acertadas ante la problemática existente.

Finalmente se llevó a cabo un seguimiento a las actividades con el fin de revisar las acciones y orientar las mismas, para beneficio de las jornadas de arborización y ornato en el fortalecimiento de la educación ambiental y el mejoramiento de la calidad de vida de los habitantes de la comunidad. 


\section{Referencias}

Bavaresco, A. (2013). Proceso metodológico en la investigación. Cómo hacer un Diseño de Investigación. Maracaibo, Venezuela: Editorial de la Universidad del Zulia.

Barros, A. (2013a,b). Geografía / Polimodal. Recursos naturales y ambientes en un mundo global. Buenos Aires: Longseller.

Chadwick, C. (1999). La psicología del aprendizaje desde el enfoque constructivista. Revista Latinoamericana de Psicología, vol. 31, núm. 3, 1999, págs. 463-475.

Contreras, J. (2016). Estrategias Ambientales para la Conservación del Bosque La Ciénaga, Ubicada en la Parroquia Barrancas, Municipio Cruz Paredes del Estado Barinas. Revista Scientific, 1(1), 72-89. Recuperado de:

https://doi.org/10.29394/scientific.issn.2542-2987.2016.1.1.5.72-89

Fernández, H. (2014a,b). Los valores ambientales en los procesos educativos Realidades y Desafío. Revista Iberoamericana sobre Calidad, Eficacia y Cambio en Educación. Volumen 7, Número 2. Rinace.

Hitcher, H. (2013). Enfoques y Principios Teóricos de la Teoría Humanista, Social y Cultural. Curso Básico para la formación de docentes. México: Editorial Trillas.

Hurtado I., \& Toro J. (2005a,b,c). Paradigmas y Métodos de Investigación en Tiempos de Cambio. 5ta Edición. Venezuela: Espíteme C.A. Recuperado de:

https://es.slideshare.net/aliriotua/paradigmas-y-metodos-deinvestigacion-autores-ivn-hurtado-len-y-josefina-toro-garrido Informe 21 (2013). Se habla mucho de él, pero... ¿Qué es el Protocolo de Kioto? Noticias en Línea. Recuperado de:

https://informe21.com/ciencia-y-tecnologia/se-habla-mucho-de-el-pero- 


\section{\%C2\%BFque-es-el-protocolo-de-kioto}

León, C. (2013). Tutela Ambiental. Caracas, Venezuela: Ediciones Paredes. Martínez, C. (2013). Valoración de áreas verdes urbanas de uso público en la comuna la Reina. Santiago: Universidad de Chile.

Montaldo, A. (2013). La Formación de valores Ambientales en la Revolución Cubana Un reto de hoy. Ponencia. La Habana, Cuba: Reunión de investigadores de la juventud.

Nebel, J. \& Wright, E. (2013). Qué es la Agenda 21. Recuperado de: http://www.ecologiaverde.com/que-es-la-agenda-21/

Red Voltaire (2006). Manifiesto de las Américas: en Defensa de la Naturaleza y la Diversidad Biológica y Cultural. Oficina Regional para América Latina y el Caribe. Página web en línea. Recuperado de: http://www.voltairenet.org/article138190.html

Sánchez, A. (2012). Plantas Ornamentales. Consejo Argentino para la Información y Desarrollo de la Biotecnología.

Sepúlveda, M. (2013). Educación Ambiental e intervención socio comunitaria. Universidad de las islas Baleares.

Tamayo \& Tamayo (2013a,b). El proceso de la Investigación científica. 4ta Edición. Limusa Noriega.

Urrea, R. (2016). Formación para la disminución de residuos sólidos, en la Escuela Básica Bolivariana "Ana de Romero". Revista Scientific, 1(1), 150-168. Recuperado de:

https://doi.org/10.29394/scientific.issn.2542-2987.2016.1.1.9.150-168

Zapata, D. (2006). Restauración de la Plaza "Delia Ávila de Zapata". Estado Sucre. Recuperado de:

http://www.monografias.com/trabajos35/plaza-avila-zapata/plaza-avilazapata.shtml 


\title{
Yosmary Del Valle Mendoza \\ e-mail: yos14mendoza@gmail.com
}

\begin{abstract}
Nacida en Venezuela. Licenciada en Educación Mención

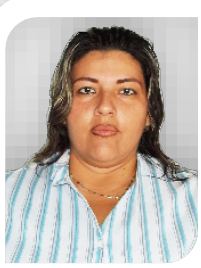

Geografía e Historia. Universidad Nacional Experimental de Los Llanos Ezequiel Zamora. Cursa estudio de Maestría en Educación Ambiente y Desarrollo en la Universidad Pedagógica Experimental Libertador, Barinas. Se desempeña como docente de aula en el Liceo Nacional Bolivariano Dr. Carlos María González Bona, Ciudad Bolivia, Municipio Pedraza. Estado Barinas durante trece años.
\end{abstract}

El contenido de este manuscrito se difunde bajo una Licencia de Creative Commons Reconocimiento- 